\title{
Communication and education for the plastic surgeon after Y2K
}

\author{
William P Graham III MD ${ }^{1}$, Donald R Mackay MD ${ }^{1}$, Paul J Gorman MD ${ }^{1}$, Lawrence L Ketch MD ${ }^{2}$ \\ ${ }^{1}$ Penn State Geisinger Health System, The MS Hershey Medical Center, Section of Plastic \& \\ Reconstructive Surgery, Hershey, Pennsylvania; ${ }^{2}$ University of Colorado Health Sciences Center, \\ Denver Colorado, USA
}

$\mathrm{W}$

ith the new millennium, how plastic surgeons educate their trainees and themselves, and communicate with each other is radically changing. With the advent of the computer and the Internet, the ways in which these processes are approached will never be the same. For communication and education, there is an obligation to multiple constituencies (Table 1). Each has its own needs, and interaction with these groups varies depending on how they interface with established plastic surgeons and leaders within academic centres. Such relationships will not be limited to the academician. As access to knowledge expands, the demands on the community surgeon will increase. A greater awareness of new developments, safer treatments, novel techniques and ways of sharing such information will be expected.

What do we owe our medical students and what may be gleaned from their education? They are the source of future trainees, and for those who choose other fields in which to practice, a potential source of referrals.

For residents and fellows, training must become relative and task oriented rather than time based. They must learn intellectual and technical competence, and be prepared for the certification process with a sound ethical foundation. With greater clinical demands placed on their faculty and greater cost of animal models, they will enter the world of virtual reality for surgical simulation.

The landscape of education and training, and the issues relative to it are shifting from the 20th to the 21 st century. The role of the physician is changing from that of a self-contained entity to that of a knowledge manager. The inability to

This article was presented as a Guest Lecture at the 53rd Annual Meeting of the Canadian Society of Plastic Surgeons, June 26, 1999, Montreal, Quebec

Correspondence and reprints: Dr William P Graham, Penn State Geisinger Health System, The MS Hershey Medical Center, Section of Plastic \& Reconstructive Surgery, PO Box 850, MC-H071, Hershey, Pennsylvania 17033, USA. Telephone 717-531-8372, fax 717-531-4339
TABLE 1

Areas of communication for plastic surgery

Students

Residents and fellows

Colleagues

Plastic surgeons

Nonplastic surgeons

Patients

Media

TABLE 2

Twenty-first century technologies available to plastic surgeons

Audiovisual interactive multimedia

Advance distribution learning

Video teletraining

Virtual surgery

assess competency will be replaced with a variety of interactive programs, from didactics to patient management and to virtual reality through surgical simulation. There are implications across the board for training, certification and credentialing.

To start the 21 st century, there are abundant resources currently operational for use in training and continuing education (Table 2). Audio visual interactive multimedia allows a graduated training situation. The degree of difficulty is matched to the user's ability, and assessment and feedback are immediately available. Advance distribution learning makes available a variety of sources, with geographic restrictions. As a cost efficient modality, it lends itself to $\mathrm{CD}$ rom or a website. Video teletraining delivers real time learning with 
TABLE 3

\section{Cornerstones for communication}

Personal commitment and interaction

Education

Reproducibility

Innovation

Efficiency

Timeliness

Highest ethical standards

two-way audio and video via satellite links; thus, any two teleconferencing sites can be bridged.

Virtual surgery, however, remains the jewel of our current endeavors. It allows immersion, interaction and feedback with reproducibility. It is able to simulate precisely the real world. The risks of training are eliminated, proficiency occurs more rapidly, costs are reduced and the dangers of cadaveric materials are eliminated.

Our interest has been to develop an anastomotic trainer for microsurgery and to evaluate its effectiveness. In our pilot study, new surgery residents began a standardized program requiring needle placement through a simulated vessel wall. By our scoring system, their abilities by day 3 after 20 mins of training daily were significantly higher than their abilities on day 1. Another study involved five surgical novices (medical students) and a needle driving task. This chore was repeated daily until a plateau was achieved at days 7 to 11 , with the group scores rising dramatically. It appears that virtual surgery will substantially reduce training time in the operative room and reduce risks to patients, yet allow appropriate progress in technical proficiency to be documented, which heretofore has been unavailable.

What does the future hold for our colleagues in plastic surgery? Obviously, the same educational tools are available for maintaining proficiency or preparing for recertification. These requirements for recertification via practice profiling, outcomes assessment and continuing education can all be part of a global program through the Web. Consultation can be done in real time across the country.

For nonplastic surgical colleagues, we must be more visible in the hospital and within local medical societies. Consultations should be a step above, with Internet references reviewed and supplied as necessary to the requesting physician. Academic colleagues can be queries and their input added.

All of these new training and educational tools, as with the airline simulators, can only lead to better care for a better informed and more demanding public. Responsible medical care must be ethical and not reside in entrepreneurialism. Imaging should be viewed as an educational and information tool, not a sales mechanism. Databases from our practices can provide updated information about techniques, cancer prevention and outcomes.

Lastly, our ability to deal with the media will allow almost instantaneous response for crisis management. Similarly, the release of new information can be more accurately disseminated at the highest ethical levels.

The cornerstones of communication for the future will still require personal commitment (Table 3). Only as long as we hold ourselves to the highest ethical standards will we maintain our credibility with our patients, our colleagues and the media. 